\title{
Theoretical Framework for Spatial Resolution in Edge-Illumination X-Ray Tomography
}

\author{
Charlotte K. Hagen, ${ }^{*}$ Fabio A. Vittoria, Marco Endrizzi, and Alessandro Olivo \\ Department of Medical Physics and Biomedical Engineering, University College London, Malet Place, London \\ WC1E 6BT, United Kingdom
}

(Received 17 July 2018; revised manuscript received 25 September 2018; published 21 November 2018)

\begin{abstract}
Edge-illumination tomography is a modality for performing $\mathrm{x}$-ray phase-contrast imaging in 3D, based on structuring the primary beam into an array of beamlets and employing a dedicated incoherent sensing mechanism. In this article, we provide a theoretical framework for the spatial resolution of this method, based on the concept of the bowtie-shaped essential support of the Radon transform in frequency space, which is well known in conventional tomography. The additional complexity caused by the use of beamlets is added to the model, and its validity is confirmed by means of simulated and experimental results. In essence, we show that the precise location of non-negligible entries in the two-dimensional frequency spectrum of an edge-illumination sinogram can be predicted by only two parameters, the beamlet width and the sample thickness. This enables the derivation of sampling schemes tailored to avoid aliasing, thus maximizing spatial resolution.
\end{abstract}

DOI: 10.1103/PhysRevApplied.10.054050

\section{INTRODUCTION}

Edge-illumination tomography $[1,2]$ is a technique for $3 \mathrm{D}$, phase-sensitive $\mathrm{x}$-ray imaging, which has been under continuous development alongside other approaches for this purpose, such as propagation-based imaging [3], analyzer-based imaging [4], grating interferometry [5], and lately speckle-based techniques [6-8]. Being able to convert the phase shift that $\mathrm{x}$-rays experience when they pass through matter into contrast, instead of, or in addition to their attenuation, has proven invaluable for samples for which attenuation contrast is inherently weak, such as soft biological tissues, or generally low- $Z$ materials. This is because for such samples phase-shift differences are often relatively larger than attenuation ones, a fact which, if properly exploited, can lead to a significant increase in contrast-to-noise ratio [9]. The various existing phaseimaging approaches differ in their experimental setup and demands on spatial and temporal coherence, and therefore their translatability to conventional (weakly coherent) $\mathrm{x}$ ray sources. Edge-illumination tomography, whose working principle is summarized below, has relatively relaxed coherence requirements and, thus, can be implemented

*charlotte.hagen.10@ucl.ac.uk

Published by the American Physical Society under the terms of the Creative Commons Attribution 4.0 International license. Further distribution of this work must maintain attribution to the author(s) and the published article's title, journal citation, and DOI. with conventional x-ray tubes, allowing a widespread use of the technique.

In brief, the method works by preshaping an x-ray beam into an array of narrow beamlets using a mask (sample mask in the following), and sensing refraction using a series of beam stops created by a second mask (detector mask in the following), as illustrated in Fig. 1. Initially, each beamlet is aligned with the edge of a beam stop, causing a specific number of photons to hit each pixel [Fig. 1(a)]. When a sample is placed into the setup (immediately downstream of the sample mask), refraction - which can be interpreted as the macroscopic manifestation of the phase shift - changes the direction of the beamlets [Fig. 1(b)]. This, in turn, causes a smaller or greater number of photons to hit the pixels, giving rise to contrast. A tomographic scan involves acquiring a set of projections while rotating the sample (forming the sinogram), and applying postprocessing methods to reconstruct cross-sectional tomograms. The period $(p)$ of the sample mask must match the demagnified pixel size, and typically the ratio between the beamlet width ( $w)$ and $p$ is within $1 / 3$ and $1 / 8$. These specific criteria require a careful design of the experimental setup. Note that, unlike in the schematic in Fig. 1, in the remainder of this paper we assume a parallel beam, implying that the sample-mask period and the pixel are both of dimension $p$, allowing the magnification between the two planes to be omitted.

Besides providing access to phase-contrast, an interesting side effect of using beamlets is that it decouples the spatial resolution from the dimensions of the detector pixels and the source, which are the driving factors for 
(a)

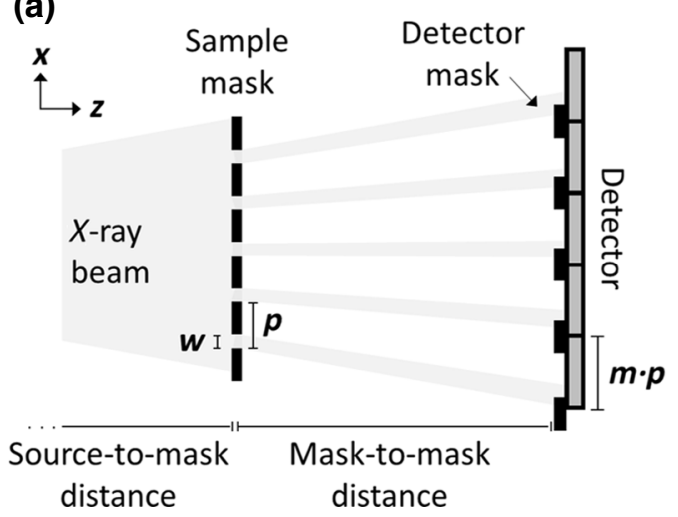

(b)

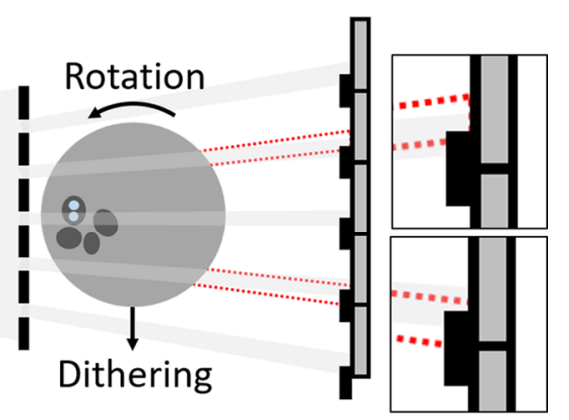

FIG. 1. Schematic illustrating the working principle of edgeillumination $\mathrm{x}$-ray tomography (note that this drawing is not to scale, shows a setup as seen from the top, and the configuration extends uniformly in the $y$ direction, which is perpendicular to the plane of the paper). this metric in conventional tomography. It has been shown that, instead, the spatial resolution is to first approximation defined by the beamlet width, provided that this is smaller than the effective (i.e., scaled to the sample plane) projected source and pixels, and that diffraction effects and any form of signal diffusion (e.g., within the detector) can be neglected [10] (for simplicity, we assume that the sample-mask plane and the sample plane coincide). Therefore, high spatial resolutions can be achieved even with relatively large pixels, as demonstrated previously when a spatial resolution of $3 \mu \mathrm{m}$ was achieved with a detector featuring $50-\mu \mathrm{m}$ pixels [11]. This also implies that a high spatial resolution is not limited to a small field of view, as the resolution is solely defined by the mask (provided that the sampling is adequate, see below), and such mask structures can readily be manufactured up to $10 \mathrm{~cm}$ by $10 \mathrm{~cm}$.

However, the use of beamlets also leads to gaps in the acquired projections, as the nonirradiated parts of the sample are not seen. As a consequence, the intrinsically high spatial resolution capabilities can only be exploited if measures are taken to fill these gaps. A common way to achieve this is by means of a process called dithering, by which the sample is scanned laterally (along the $x$ direction) in steps smaller than the sample-mask period, a frame is acquired at each step, and all frames are subsequently combined into a single, upsampled projection. Dithering can be performed either in step-and-shoot or continuous mode; in the former, the sample is displaced in between the acquisition of consecutive frames (i.e., while the detector is reading out), whereas in the latter the sample is displaced during the acquisition of frames (i.e., while the detector is integrating) [12]. While the effect of dithering on spatial resolution has previously been analyzed for two-dimensional (2D) (planar) edge-illumination imaging [10], an investigation for three-dimensional (3D) (tomographic) imaging, where sinograms are acquired rather than individual projections, is still lacking. In tomography, dithering is combined with the rotation of the sample, involving the sampling of lateral and angular coordinates, making their interaction more complex.
In conventional tomography (i.e., when the beam is not structured and the setup is only sensitive to the attenuation of x-rays), the interplay between lateral and angular sampling, and their effect on the spatial resolution in the tomograms, is well understood thanks to a theory by Rattey and Lindgren [13]. Their work provides a simple but sufficiently accurate description of how a tomographic imaging system responds in frequency space to an impulse it receives in real space; in fact, it has been demonstrated that the spectral content of the Radon transform is confined to a well-defined area that has the shape of a bowtie. This has proven to be a powerful insight, not only for characterizing the spatial resolution in tomographic scanners in general, but also for, e.g., improving the image reconstruction process [14] and developing new approaches to region-of-interest scanning [15].

Here, we apply the concepts by Rattey and Lindgren to enhance the understanding of spatial resolution in edgeillumination x-ray tomography, and how it is linked to the sampling of the sinogram along the lateral and angular directions. Due to the beamlet-based decoupling of spatial resolution from the pixel and source dimensions, this relationship is expected to differ from that in conventional tomography. In Sec. II, we provide an appropriate adaptation of the theory, which requires treating the cases of step-and-shoot and continuous dithering separately. We then exploit this theory to derive optimal sampling conditions that maximize spatial resolution while minimising dose. In Sec. III, we present a series of simulated and experimental results that validate the presented framework. This article concludes with a brief summary and a critical evaluation of the work.

\section{THEORY}

The spatial resolution in a tomogram is generally defined by a combination of the following aspects: the highest spatial frequency that is present in the imageformation process, the way by which the data are sampled, and any postprocessing applied to the data, e.g., interpolation, filtering, or tomographic reconstruction. In 
the following, we will discuss the first two aspects in the context of edge-illumination tomography, but will neglect the latter aspect, as the multitude of available postprocessing methods would go beyond the scope of this article.

In conventional tomography, it is common that the spatial resolution in a 3D volume is nonisotropic; typically, the in-slice resolution (relating to cross sections perpendicular to the axis of rotation) exceeds the slice thickness (relating to the direction parallel to the axis of rotation). This is done to keep the dose within acceptable limits, as an increase of spatial resolution translates into an increase in dose. In the case of edge-illumination tomography, it is sensible to adopt this distinction, as the shape and orientation of the beamlets shown in Fig. 1 mean that only the in-slice resolution is decoupled from the pixel and source dimensions, while the slice thickness remains defined by the pixel size [10]. In this article, we therefore focus on analyzing the effect of sampling on the in-slice resolution. Note that a differently shaped sample mask, e.g., one that creates an array of pencil beams, would decouple also the slice thickness from the pixel size.

\section{A. The 2D frequency response of edge-illumination x-ray tomography}

It is generally useful to discuss spatial resolution in both real and frequency space, which are related via the Fourier transform. Understanding how an imaging system responds in frequency space to an impulse it receives in real space provides an understanding of the highest spatial frequency that is involved in the image-formation process, and, thus, of the spatial resolution that can be accessed (in imaging systems' theory, this is often described by means of the modulation transfer function).

One way of describing the frequency response of a tomographic system is by means of the Fourier transform of the Radon transform, i.e., the mathematical description of the sinogram. As stated above, for conventional tomography it has been shown [13] that, when a sample is $b$-bandlimited, i.e., it can be described by a function whose Fourier transform is confined to a disc of radius $b$ around the origin of frequency space, the Fourier transform of its Radon transform is confined to the set of spatial frequencies $\left(\omega_{\theta}, \omega_{x}\right)$ fulfilling $\left|\omega_{\theta}\right|<\max \left(1,1+\left|\omega_{x}\right| t / 2\right)$ and $\left|\omega_{x}\right|<b$. Here, $t$ denotes the thickness of the sample, which for samples of noncylindrical shape refers to their thickest part. This set of spatial frequencies, known as the Radon transform's essential support, has the shape of a bowtie. Although the notion of a bandlimited sample is a theoretical construct, an actual bandlimit is imposed by the highest spatial frequency that the imaging system can transfer. In conventional tomography, where the sample is illuminated by the full $\mathrm{x}$-ray beam, this is defined by the pixel response function (under the assumption that the effect of the source blur can be neglected), which, in the idealized case, can be described by a box function of width $p$. This becomes a sinc function in frequency space, with a first root at $1 / p$ (assuming the unitary, ordinary frequency convention of the Fourier transform) and insignificant magnitude for spatial frequencies larger than that. Thus, the actual bandlimit imposed by the imaging system is approximately $1 / p$.

This is somewhat more complex in edge-illumination tomography, and requires treating the cases of step-andshoot and continuous dithering separately. In the former, the imposed bandlimit is given by the inverse of the beamlet width, $1 / w$. This is because, analogously to the pixel in conventional tomography, the beamlet can be described by a box function (of width $w$ ) in real space, which becomes a sinc function (with the first root at $1 / w$ ) in frequency space. When continuous dithering is applied, the sample motion acts as an additional low-pass filter, thus changing the transferred frequency content. Assuming that the sample is moved by a lateral distance of $a$ during the acquisition of a frame, the system's response in real space can be modeled as the convolution of a box function (of width $a$ ) with the beamlet. When $a<w$, the frequency response is still dominated by the beamlet width, thus not changing the bandlimit compared to the step-and-shoot case. However, when $a>w$, the sample motion becomes the dominating factor, narrowing the frequency response and reducing the bandlimit to approximately $1 / a$. This is illustrated in Fig. 2(a) for $a=0$ (corresponding to step-and-shoot dithering), $a=2 w$ and $a=p$. Figure 2(b) compares the frequency response of the latter case $(a=p)$ to that of conventional tomography where the full x-ray beam is used instead of beamlets. While there are some small differences in the higher spatial frequencies, the bandlimit (approximately given by the first root of the response function) is the same in both cases. This shows that edgeillumination tomography can be used to reproduce the frequency response of a conventional tomography scanner by applying continuous dithering and moving the sample by a distance equal to the pixel size.

The option to choose between step-and-shoot and continuous dithering, and thereby to manipulate the accessible frequency content, implies that edge-illumination $\mathrm{x}$-ray tomography exhibits some degree of flexibility in terms of spatial resolution, with resolutions ranging from $p$ (as defined by the sample-mask period) down to $w$ (as defined by the beamlet width) accessible. This is another competitive advantage of the technique, as the spatial resolution can be tailored to the needs of the imaged sample, also while using the same experimental setup. For example, as continuous dithering with a relatively large sample movement leads to a dose reduction (in a similar fashion as increasing the pixel size does in conventional tomography), this approach may be favorable for radiation-sensitive samples. 
(a)

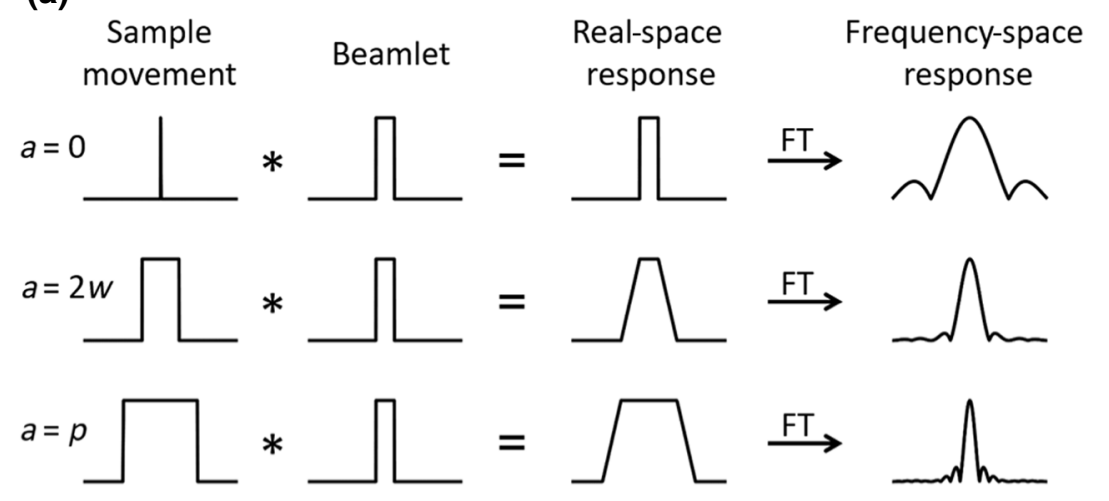

(b)

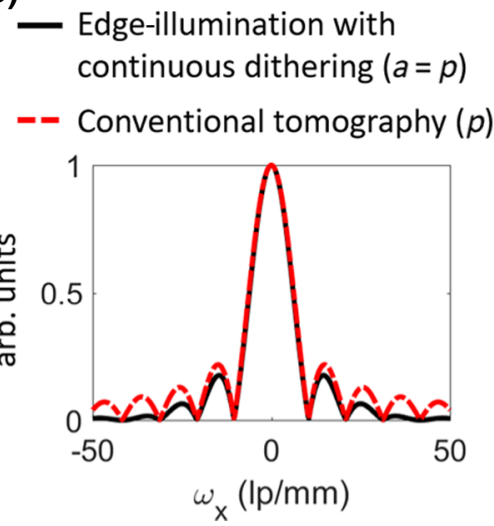

FIG. 2. (a) The effect of moving the sample during the acquisition of a frame when applying continuous dithering. The sample movement, which can be modeled by a box function of width $a$, is convolved with a box function of width $w$ describing the beamlet. This corresponds to applying a low-pass filter, modulating the frequency response of the imaging system. FT, Fourier transform. (b) Comparison of the frequency response of the edge-illumination method when the sample is moved by a distance equal to the pixel size during continuous dithering to that of conventional tomography with the same pixel size.

Having established the bandlimit determined by the imaging setup, we can now describe the essential support of the Radon transform of edge-illumination tomography in frequency space for step-and-shoot [Eq. (1)] and continuous dithering [Eq. (2)]. For comparison, the essential support of conventional tomography is given by Eq. (3).

$$
\begin{gathered}
\left(\omega_{\theta}, \omega_{x}\right) \quad \text { fulfilling } \quad\left|\omega_{\theta}\right|<\max \left(1,1+\frac{\left|\omega_{x}\right| t}{2}\right) \\
\text { and }\left|\omega_{x}\right|<\frac{1}{w}, \\
\left(\omega_{\theta}, \omega_{x}\right) \quad \text { fulfilling }\left|\omega_{\theta}\right|<\max \left(1,1+\frac{\left|\omega_{x}\right| t}{2}\right) \\
\text { and }\left|\omega_{x}\right|<\min \left(\frac{1}{w}, \frac{1}{a}\right), \\
\left(\omega_{\theta}, \omega_{x}\right) \quad \text { fulfilling }\left|\omega_{\theta}\right|<\max \left(1,1+\frac{\left|\omega_{x}\right| t}{2}\right) \\
\text { and }\left|\omega_{x}\right|<\frac{1}{p} .
\end{gathered}
$$

The corresponding areas of 2D frequency space are shown in Figs. 3(a) and 3(b) next to the respective imaging setup, highlighting again that edge-illumination x-ray tomography provides access to higher spatial frequencies than a conventional tomography system using the same size pixels.

\section{B. Optimal sampling conditions for edge-illumination x-ray tomography}

In general, the process of sampling can be modeled as the multiplication of an ideal signal with a Dirac comb.
In frequency space, this multiplication corresponds to a convolution between the Fourier transforms of that signal and Dirac comb, yielding shifted replicas of the former. Any overlap between the replicas causes aliasing, i.e., an incorrect weight to be assigned to the affected spatial frequencies. This observation leads to Nyquist's theorem for $b$-bandlimited signals $[16,17]$ : in order to avoid aliasing, the shifted replicas must be separated by a distance of at

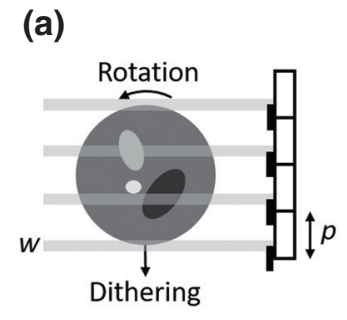

(b)

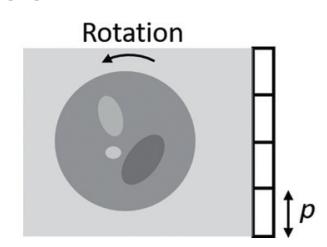

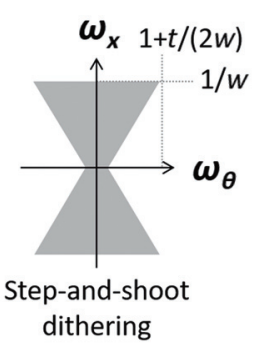
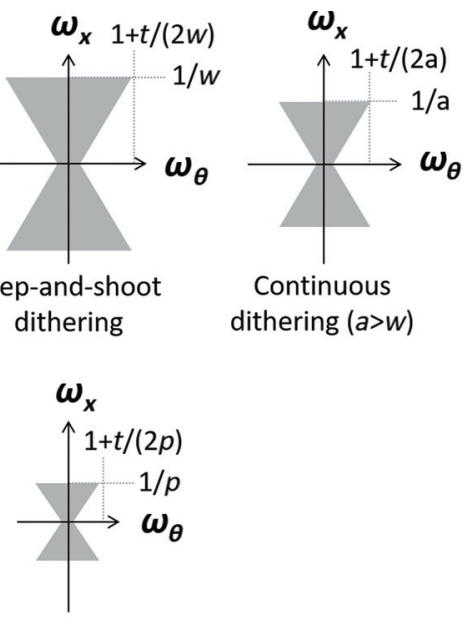

FIG. 3. (a) Simplified schematic of edge-illumination x-ray tomography with pixel size $p$ and beamlet width $w$, and the associated bowtie-shaped essential supports of the Radon transform when applying step-and-shoot dithering (middle) and continuous dithering with a sample motion by distance $a(a>w)$ during the acquisition of each frame (right). In the lateral direction, these are bandlimited by $1 / w$ and $1 / a$, respectively. (b) Schematic of a conventional tomography setup with pixel size $p$ and the associated essential support of the Radon transform, which is bandlimited in the lateral direction by $1 / p$. 
TABLE I. Overview of optimal sampling conditions for edge-illumination x-ray tomography.

\begin{tabular}{lcccc}
\hline \hline Dithering mode & Sampling scheme & $\begin{array}{c}\text { Lateral sampling } \\
\text { interval }\left(\Delta_{x}\right)\end{array}$ & $\begin{array}{c}\text { Angular sampling } \\
\text { interval }\left(\Delta_{\theta}\right)\end{array}$ & $\begin{array}{c}\text { Angle-dependent } \\
\text { offset }(d)\end{array}$ \\
\hline Step-and-shoot & Rectangular & $w / 2$ & $1 /(2+t / w)$ & 0 \\
Step-and-shoot & Interlaced & $w$ & $1 /(4+t / w)$ & $w / 2$ \\
Continuous $(a>w)$ & Rectangular & $a / 2$ & $1 /(2+t / a)$ & 0 \\
Continuous $(a>w)$ & Interlaced & $a$ & $1 /(4+t / a)$ & $a / 2$ \\
\hline \hline
\end{tabular}

least $2 b$, which can be achieved by sampling in real space with an interval of $1 /(2 b)$. Nyquist's theorem can easily be extended to two dimensions, by multiplying a 2D signal with a Dirac brush (i.e., a 2D Dirac comb), and noting that the convolution in Fourier space yields shifted tiles instead of one-dimensional (1D) replicas, which again must be spaced apart by a sufficient distance so as to not overlap. The process of sampling the Radon transform during a tomographic scan can therefore be thought of as creating shifted replicas of its essential support, i.e., as tiling frequency space with bowties. The Dirac brush applied during a tomographic scan (i.e., the real-space-sampling grid) can be described by vectors $a_{1}=\left(\Delta_{\theta}, 0\right)$ and $a_{2}=$ $\left(0, \Delta_{x}\right), 0<\Delta_{x} \leq p$, where $\Delta_{\theta}$ and $\Delta_{x}$ are the angular and lateral sampling interval, respectively. The corresponding frequency-space sampling grid can be described by vectors $b_{1}=\left(1 / \Delta_{\theta}, 0\right)$ and $b_{2}=\left(0,1 / \Delta_{x}\right)$. The lengths of these vectors determine the spacing between the shifted bowties, allowing conditions for a no-overlap scenario to be formulated.

To derive such optimal sampling conditions for edgeillumination x-ray tomography, it is again necessary to treat the cases of step-and-shoot and continuous dithering separately. In the case of the former, a no-overlap scenario is achieved when $\left|b_{1}\right|=2+t / w$ and $\left|b_{2}\right|=2 / w$, which corresponds to lateral and angular sampling intervals of $\Delta_{x}=w / 2$ and $\Delta_{\theta}=1 /(2+t / w)$ (note that $\Delta_{\theta}$ is given in radians). In practice, this means that the sample must be laterally displaced $2 p / w$ times by a distance of half the beamlet width and a frame taken at each displacement, a procedure which is to be repeated at every rotation angle. Thus, this sampling scheme requires the acquisition of $\left(p / \Delta_{x}\right) \cdot\left(\pi / \Delta_{\theta}\right)=(2 p / w) \cdot[\pi(2+t / w)]$ frames, assuming a total range of $180^{\circ}$ for the sample rotation. Analogously, when continuous dithering is applied (with $a>w$ ), the shifted bowties must be spaced apart by at least $\left|b_{1}\right|=2+t / a$ and $\left|b_{2}\right|=2 / a$, which corresponds to $\Delta_{x}=a / 2$ and $\Delta_{\theta}=1 /(2+t / a)$. As the required $\Delta_{x}$ is smaller than the distance $a$, in practice this means that the sample must be shifted back by $a / 2$ after the acquisition of each frame (during which the sample is moved laterally by distance $a$ ). The number of frames required for continuous mode dithering is thus $(2 p / a) \cdot[\pi(2+t / a)]$. These sampling conditions, which are summarized in the first and third rows of Table I, are optimal in the sense that, to first approximation, no increase in spatial resolution can be achieved by further decreasing the lateral or angular sampling intervals.

The vectors $a_{1}, a_{2}, b_{1}$, and $b_{2}$ as defined above span rectangular grids, leading to an orthogonal arrangement of bowties in frequency space [Figs. 4(a)-4(c)]. However, as was already observed for conventional tomography [13, $18,19]$, an alternative tiling that better accommodates the actual shape of the bowties can be achieved by sampling on interlaced grids [Figs. 4(d)-4(f)]. These can be described by vectors $a_{1}=\left(\Delta_{\theta},-d\right)$ and $a_{2}=\left(\Delta_{\theta}, \Delta_{x}-\right.$ $d)$ in real space, and $b_{1}=\left[1 / \Delta_{\theta}\left(1-d / \Delta_{x}\right),-1 / \Delta_{x}\right]$ and $b_{2}=\left(d / \Delta_{\theta} \Delta_{x}, 1 / \Delta_{x}\right)$ in frequency space, where $d$ denotes an offset between adjacent rows and columns of grid points. Edge-illumination x-ray tomography possesses the necessary flexibility for realizing this experimentally, as it provides the option to offset the sample at every other rotation angle with respect to the lateral sampling step, allowing the interlaced geometry to be implemented in a straightforward fashion. When sampling on interlaced grids and employing step-and-shoot dithering, a lateral sampling interval of $\Delta_{x}=w$, a relative offset of $d=w / 2$, and an angular sampling interval of $\Delta_{\theta}=1 /(4+t / w)$ are required to generate a no-overlap tiling of frequency space. In practice, this requires the sample to be displaced laterally $p / w$ times by a distance equal to the width of the beamlet at each tomographic view, and, at every other view, the sample to be displaced laterally by half the beamlet width. This scheme requires the acquisition of $(p / w)[\pi(4+t / w)]$ frames, which is approximately two times less than what is required when sampling on rectangular grids. Thus, these sampling conditions are not only optimal in terms of spatial resolution, but also in terms of dose as they achieve the maximum spatial resolution with the minimum amount of data. The analogous, doseoptimized sampling conditions for continuous dithering are summarized in the bottom row of Table I. Note that the reconstruction of tomographic images from data sampled on interlaced grids requires the use of specialized algorithms [20].

An interlaced acquisition scheme has previously been suggested in the context of phase tomography for grating interferometry [21]. However, that method is fundamentally different to the interlaced sampling discussed here, as it focuses on phase stepping, a procedure by which one of the gratings is scanned with respect to the other in order to quantitatively extract the phase shift. Interlaced phase 
(a)

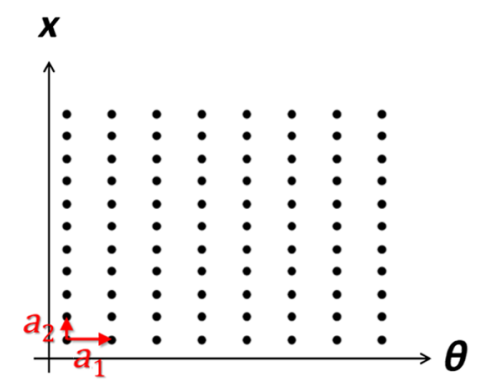

(d)



(b)

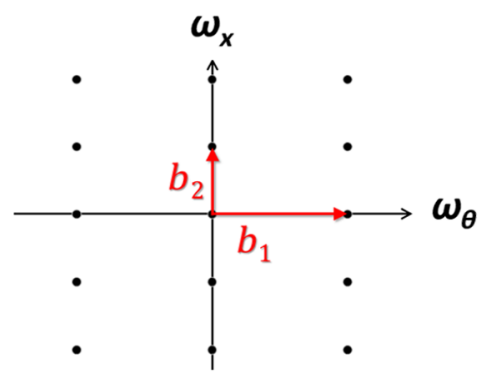

(e)



(c)

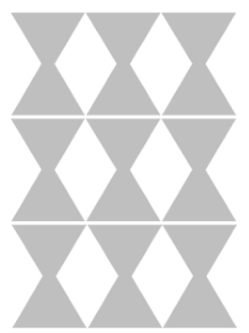

(f)



FIG. 4. Different possible sampling schemes for edgeillumination x-ray tomography: (a) rectangular real space grid, (b) rectangular frequency space grid, (c) the corresponding orthogonal arrangement of the Radon transform's essential support in frequency space, (d) interlaced real-space grid, (e) interlaced frequency-space grid, (f) the corresponding interlaced arrangement of the Radon transform's essential support in frequency space. stepping, during which the sample is slightly rotated in between phase steps, has been shown to reduce artefacts typically associated with limited field-of-view tomography; however, it does not have any effect on spatial resolution.

\section{VERIFICATION THROUGH SIMULATIONS AND EXPERIMENTS}

In order to validate the presented theoretical framework for spatial resolution in edge-illumination tomography, a series of simulation-based and experimental studies are performed. Experimental data are acquired with a setup featuring a Rigaku 007 HF MicroMax (Japan), a rotating anode (Mo) x-ray tube with a horizontal focal spot of approximately $70 \mu \mathrm{m}$. The source, when operated at $25 \mathrm{~mA}$ and $40 \mathrm{kVp}$ (no external filtration), produces a polychromatic spectrum with a mean energy of approximately $18 \mathrm{keV}$. The detector is Pixirad-2 (Italy), a single photon counter with a pixel size of $62 \mu \mathrm{m}$. The sample mask has a period $(p)$ of $48 \mu \mathrm{m}$, and its aperture size, which defines the width of the beamlets $(w)$, is $12 \mu \mathrm{m}$. The period and apertures of the detector mask are 60 and $15 \mu \mathrm{m}$, respectively. Both masks are fabricated by electroplating gold strips onto a graphite substrate (Creatv Microtech, USA). The source-to-mask and mask-to-mask distances are 1.6 and $0.4 \mathrm{~m}$, respectively, corresponding to a magnification factor of $m=1.25$ between the two planes. The detector is placed approximately $6 \mathrm{~cm}$ downstream of the detector mask to ensure the pixel size matches the projected periods of the masks. The scanned sample is a custom-made phantom composed of several plastic wires (diameter $=300 \mu \mathrm{m})$ arranged in a plastic cylinder (diameter $=3.2 \mathrm{~mm}$ ).

Simulations are based on a wave optics model (based on the wave theory of optics in the Fresnel approximation), which has previously been found to reliably predict experimental edge-illumination results [22]. The parameters for the simulation are chosen to match the setup described above; however, a monochromatic beam with an energy of $18 \mathrm{keV}$ is considered for the sake of simplicity. As previously demonstrated [23], an edge-illumination setup responds to different energies in the same way (the energy dependence of the signal is due to the sample alone), and hence this assumption does not affect the results of our analysis. We simulate scans of a numerical resolution phantom consisting of ten radially arranged sets of black and white wires with negative and positive attenuation and refraction properties, providing a visual representation of the line pairs per $\mathrm{mm}(\mathrm{lp} / \mathrm{mm})$ definition of spatial resolution. The diameters of the wires are approximately 83, $71,62.5,56,50,25,17,12.5,10$, and $8 \mu \mathrm{m}$, corresponding to $6,7,8,9,10,20,30,40,50$, and $60 \mathrm{lp} / \mathrm{mm}$. Such a sample could be realized experimentally by placing two types of wires in a surrounding liquid with attenuation and refraction properties in between the ones of the wires. Please note that the fact that we use a much simpler phantom for our experimental results does not affect the overall conclusions of the paper. Rather, it reflects the sampleindependent nature (apart from relying on its thickness) of the theoretical model.

For both simulation and experiment, tomographic reconstruction is performed by means of the filtered backprojection algorithm. To make this possible, all data are 
sampled on rectangular grids (the reconstruction from interlaced grids will be investigated as part of future work). Due to the fact that edge-illumination tomography provides phase-contrast (in the form of refraction) in addition to attenuation contrast, a phase-retrieval algorithm is applied prior to reconstruction, converting refraction, which manifests as dark and bright fringes at contours and boundaries within the sample, into area signal. We apply a recently developed single-shot method [25] that takes only one projection as the input at each tomographic angle (other methods require two or more input projections [24]). The method, which is an adaptation of Paganin's approach [26] to edge-illumination tomography, assumes a linear relationship between refraction and attenuation, which is strictly valid only for quasihomogeneous samples, i.e., when the ratio between attenuation and refraction properties is constant, which is fulfilled for all samples involved in this study.

As a first step, we use a combination of simulated and experimental results to verify the existence of the essential support of the Radon transform in frequency space for edge-illumination tomography. By means of simulation, we generate a sinogram of the numerical phantom in order to then analyze its 2D frequency spectrum. Considering that the overall thickness of the numerical phantom (the distance from the origin at which the wires are placed, plus the radius of the thickest wires) is $t=1 \mathrm{~mm}$, in addition to the beamlet width $(w=12 \mu \mathrm{m})$ of the simulated setup, the optimal lateral and angular sampling intervals (according to Table I for step-and-shoot dithering and rectangular grids) are $\Delta_{x}=6 \mu \mathrm{m}$ and $\Delta_{\theta}=0.0117 \mathrm{rad}$ (= $\left.0.6714^{\circ}\right)$. To exclude any undersampling artefacts, the simulation is initially run with $\Delta_{x}$ and $\Delta_{\theta}$ ten times smaller than these conditions. The results of the simulation are shown in the top row of Fig. 5. Panel (a) shows the reconstructed tomogram, providing a visual representation of the numerical phantom, and (b) shows the sinogram. While phase retrieval is applied prior to the reconstruction of the tomogram, the sinogram still contains a combination of attenuation and phase (refraction) contrast. Panel (c) shows the sinogram's 2D Fourier transform, with the vertical and horizontal spatial frequency axes relating to the lateral and angular directions, respectively. Note that sampling at lateral and angular intervals ten times smaller than the optimal ones provides access to a very large area of Fourier space (much larger than the anticipated essential support), so in the interest of clarity only the relevant part of Fourier space is shown. The red dashed line indicates the essential support in frequency space that is predicted by the theory for a sample of the given thickness and a setup with the given beamlet width [Eq. (1)]. The match between theory and simulation is evident, as virtually the entire frequency information is located precisely within the predicted region.

The bottom row of Fig. 5 shows the corresponding experimental data obtained for the scanned wire phantom (note this is acquired over 360 degrees, although only a $180^{\circ}$ region of the sinogram is shown). For practical reasons (time constraints), it is not possible to sample at intervals as high as in the simulated case, and the applied lateral and angular sampling steps are $\Delta_{x}=6.86 \mu \mathrm{m}$ and $\Delta_{\theta}=0.0044 \mathrm{rad}\left(=0.25^{\circ}\right)$. These sampling intervals
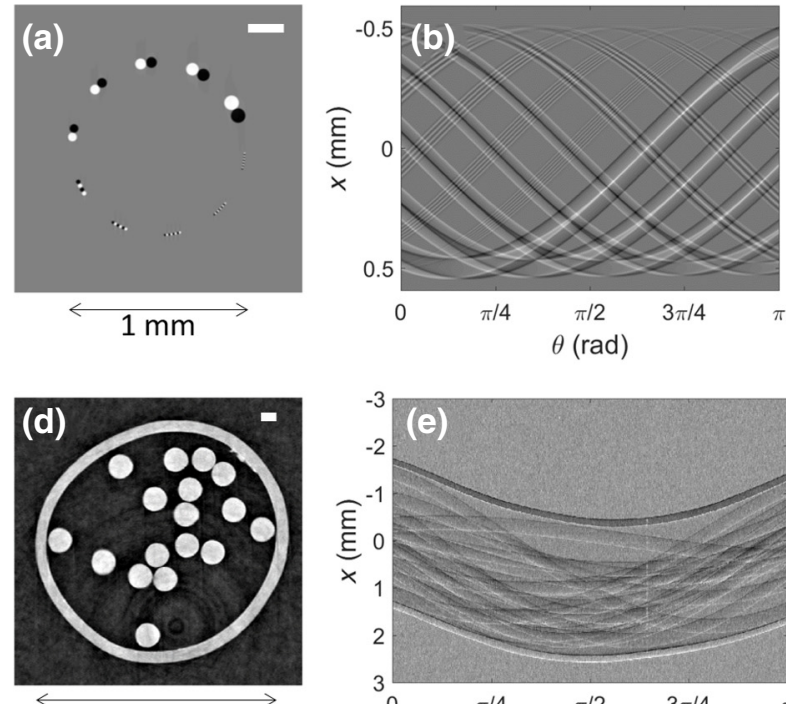

$3.2 \mathrm{~mm}$

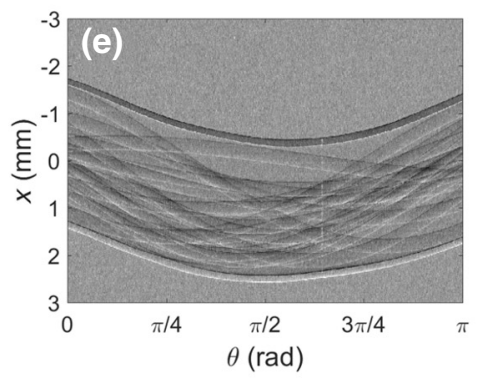

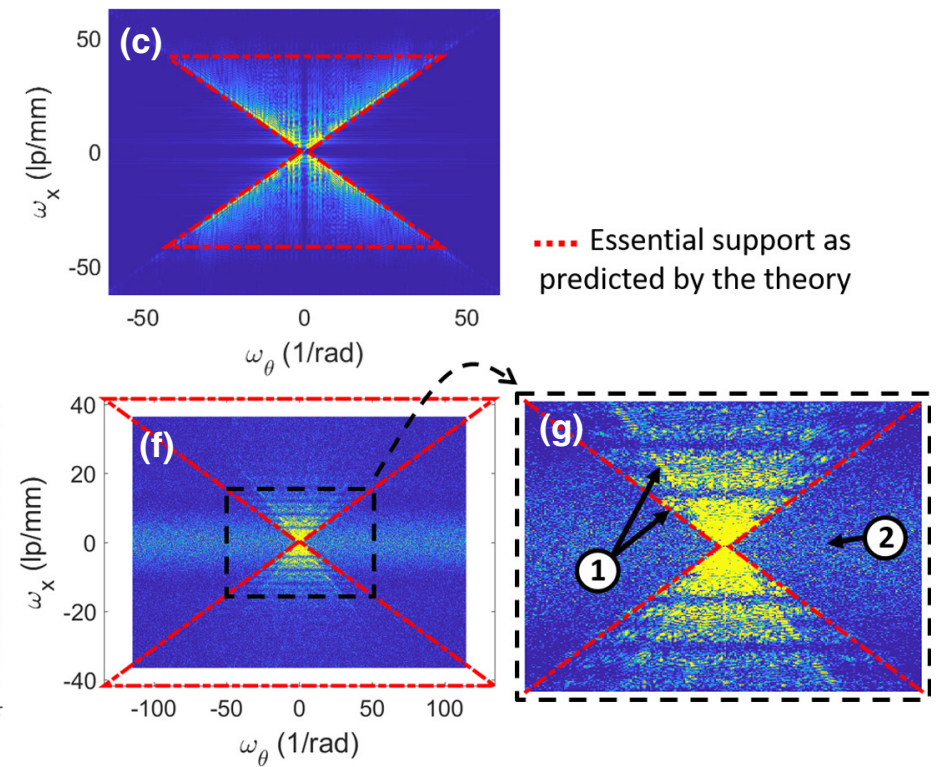

FIG. 5. Simulated data of the numerical resolution phantom (top row) and experimental data of the custom-built wire phantom (bottom row): tomogram reconstructed from the simulated sinogram (scale bar, $200 \mu \mathrm{m}$ ) (a), simulated sinogram (b), 2D Fourier transform of the simulated sinogram (c), tomogram reconstructed from the experimental sinogram (scale bar, $200 \mu \mathrm{m})$ (d), experimental sinogram (e), 2D Fourier transform of the experimental sinogram (f), and enlarged central area (g). 
require that seven frames are acquired at each rotation angle. The overhead per frame (i.e., the time additional to the actual $\mathrm{x}$-ray exposure of $1 \mathrm{~s}$, required for detector read out and motor movements) is approximately 4 $\mathrm{s}$, though this has not been optimized. Note that these intervals fall slightly short on the optimal sampling conditions for a sample with $t=3.2 \mathrm{~mm}$ and the given imaging setup (according to Table I, the optimal steps in this case are $\Delta_{x}=6 \mu \mathrm{m}$ and $\Delta_{\theta}=0.0037 \mathrm{rad}$ ), implying that the accessible region of frequency space is smaller than the theoretical bowtie support. Nevertheless, within the available region, the match between experiment and theory is evident, as shown by the enlarged area in (g).

Despite the overall agreement with the theory, two differences between the experimental and the simulated 2D frequency spectra can be observed, indicated by the numbered arrows in Fig. 5(g); (1) the experimental one features a second, inner bowtie in addition to the outer one that aligns with the theoretical prediction, and (2) a horizontal band of background signal is present. Point (1) is a result of the custom-built experimental phantom effectively being composed of several subsamples of different thicknesses (i.e., each wire located at a different distance from the samples centre can be considered a subsample). Since the Radon transform and Fourier transform are linear operations, each subsample is expected to generate its own bowtie. To support this explanation, we add a layer of wires to the numerical phantom closer to its centre and repeated the simulation (now using the optimal sampling conditions). The results are shown in Figs. 6(a) and 6(b). As expected, the sinogram's 2D Fourier transform shows a second, inner bowtie, analogous to that observed experimentally. Point (2) in Fig. 5(g) can be attributed to the processing of the experimental data. To compensate for system drifts over time, a flat field is collected at each angular view and used to correct all frames (i.e., the different lateral sampling positions) taken at that angle. While this is an efficient method to eliminate ring artefacts, it also correlates the noise between the individual frames (since they are divided by the same noisy flat field), causing the observed horizontal band. To support this explanation, we process the experimental data again without flat field correction. The results are shown in Figs. 6(c)-6(e); as can be seen, the horizontal band of background signal is eliminated from the sinogram's 2D frequency spectrum; however, strong ring artefacts are created in the reconstructed tomogram at the same time.

As a second step, we analyze the validity of the optimal sampling conditions by considering the case of step-andshoot dithering and rectangular grid sampling as an example. For this purpose, we simulate scans of the numerical phantom with lateral and angular sampling intervals two times larger than, equal to, and two times smaller than the optimal ones. We then analyze the spatial resolution in each respective reconstructed tomogram by fitting an error function to an edge profile yielding the edge spread function (ESF), calculating its derivative to obtain the line spread function (LSF), and measuring its FWHM. The results are shown in Fig. 7. In accordance with the theory, the optimal sampling conditions [Fig. 7(b)] lead to a measured spatial resolution of $12 \mu \mathrm{m}$ (approximately equal to $42 \mathrm{lp} / \mathrm{mm}$ ), equal to the beamlet width. This is reflected
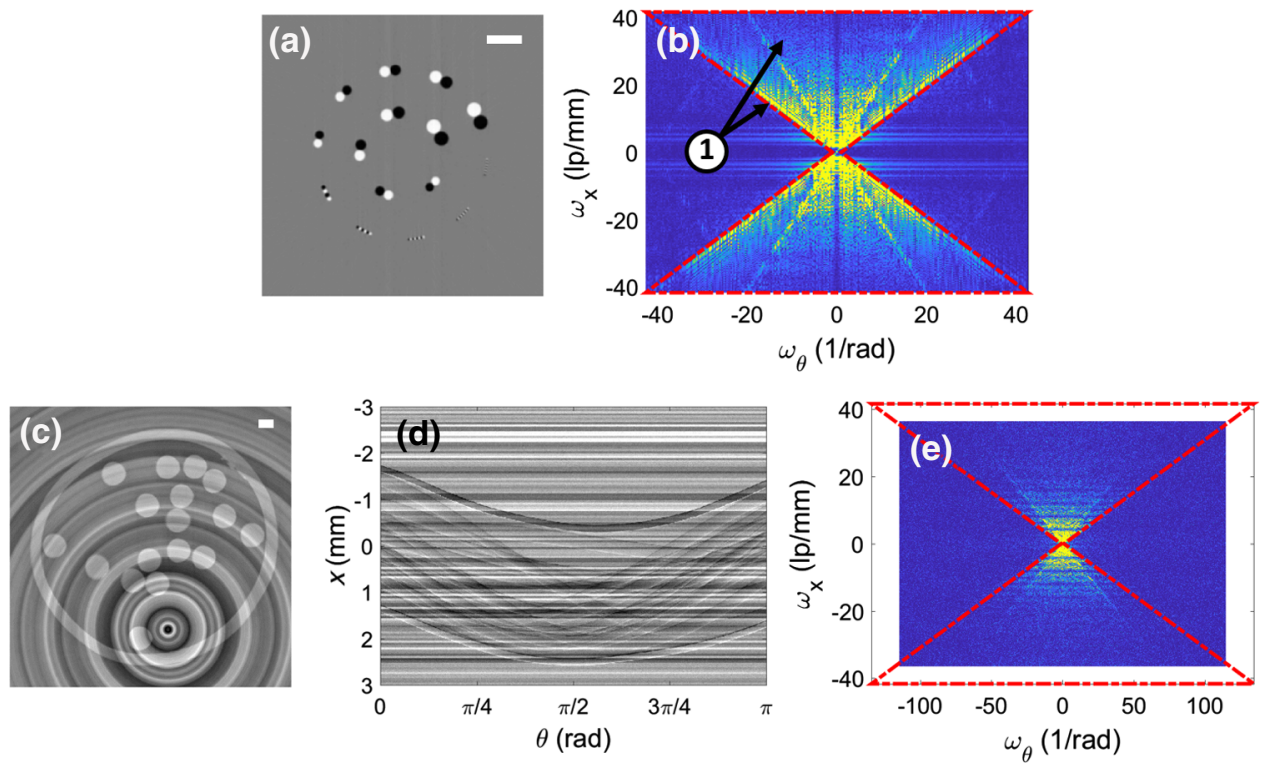

FIG. 6. Simulated (top row) and experimental results (bottom row) generated to explain observations (1) and (2) in Fig. 5(f). (a) Simulated tomogram of the updated numerical phantom, now containing additional sets of wires (scale bar, $200 \mu \mathrm{m}$ ), (b) 2D Fourier transform of the simulated sinogram. (c) Experimental tomogram (scale bar, $200 \mu \mathrm{m}$ ) and (d) corresponding sinogram after eliminating flat fielding from the data processing, (e) 2D Fourier transform of the experimental sinogram. 

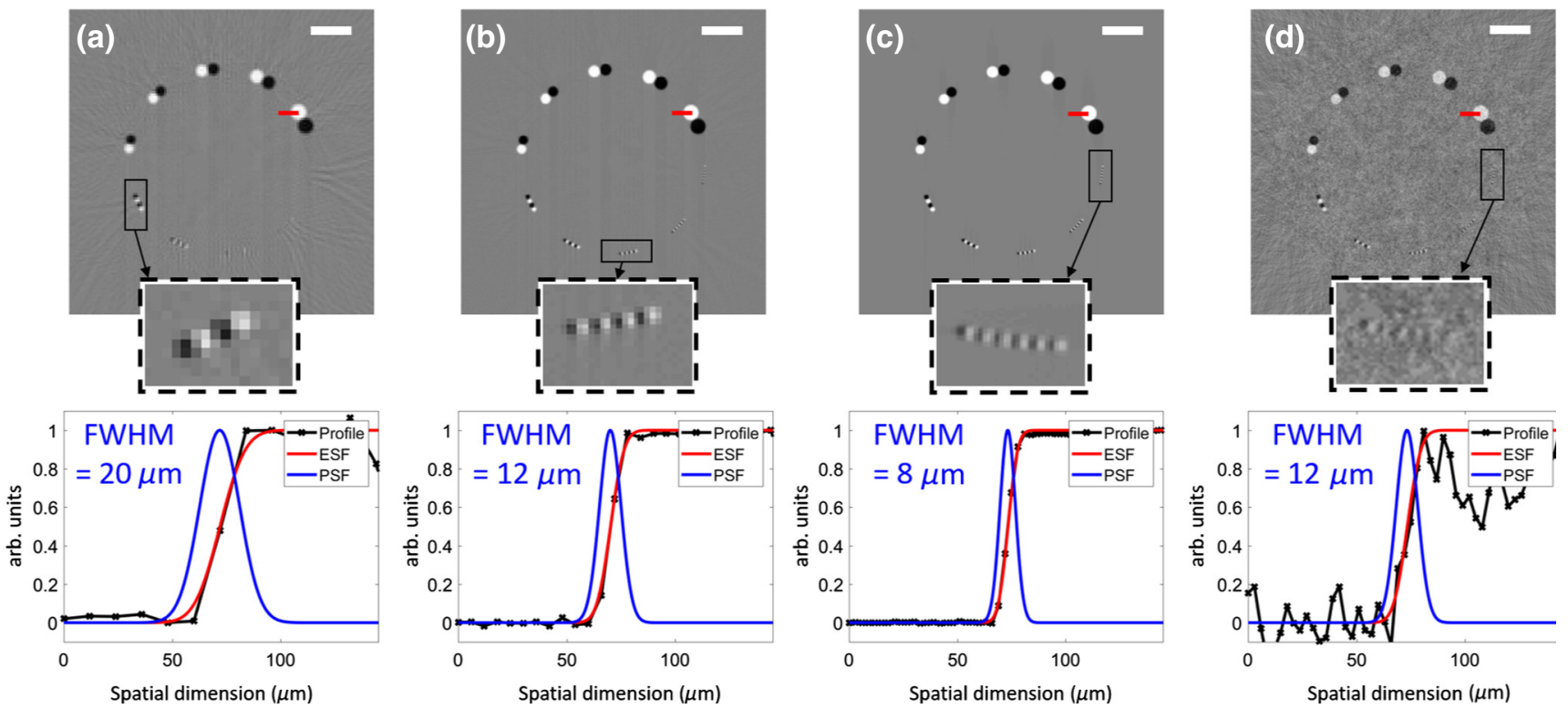

FIG. 7. Tomograms of the numerical resolution phantom simulated with different lateral and angular sampling intervals and corresponding spatial resolution analysis (all scale bars, $200 \mu \mathrm{m}$ ). (a) Double the optimal intervals, (b) optimal sampling (according to the first row of Table I), (c) half the optimal intervals, (d) half the optimal intervals with added noise.

by the small inset, which shows an enlargement of the 40 $\mathrm{lp} / \mathrm{mm}$ wires, clearly allowing adjacent black and white circles to be differentiated. When selecting lateral and angular sampling steps larger than the optimal ones [Fig. 7(a)], the measured spatial resolution is reduced as expected $(20$ $\mu \mathrm{m}=25 \mathrm{lp} / \mathrm{mm}$; the inset shows an enlargement of the 20 $\mathrm{lp} / \mathrm{mm}$ wires). While, according to the theory, we would expect that sampling with smaller intervals than the optimal ones [Fig. 7(c)] would provide no resolution gain beyond the beamlet width, we measure a spatial resolution of $8 \mu \mathrm{m}(=62.5 \mathrm{lp} / \mathrm{mm})$ in this case. This high resolution is also reflected by the inset, clearly revealing wires at 60 $\mathrm{lp} / \mathrm{mm}$.

To explain this discrepancy between simulation and theory, it is important to note that the assumption made to derive the latter (that the highest transmitted frequency in edge-illumination $\mathrm{x}$-ray tomography is determined by the inverse of the beamlet width, $1 / w$ ), is not accounting for higher-order contributions that can be observed in the absence of noise (as is the case in our simulation). To show this, we analyze the 2D Fourier spectrum of the sinogram leading to the results in Fig. 7(c) in more detail. As presented in Fig. 8, this reveals the presence of information outside the bowtie-shaped frequency-space essential support (enlarged and contrast adjusted in the inset); these entries are, however, approximately two orders of magnitude smaller than those within the bowtie. The presence of these higher-order frequencies can be due to several factors, most prominently the ripples of the sample-mask apertures' frequency response function beyond its first roots (shown in Fig. 2). These are typically ignored as entries at these frequencies are often below the noise level in experimental data. To demonstrate this, we add white Gaussian noise (power, $-30 \mathrm{~dB}$ ) to the sinogram yielding the results in Fig. 7(c), reconstruct the tomogram again, and repeat the resolution analysis. The results, shown in Fig. 7(d), now reveal a measured resolution of $12 \mu \mathrm{m}$, matching the theory.



FIG. 8. 2D Fourier transform of the sinogram simulated for the numerical phantom with half the optimal lateral and angular sampling intervals. 


\section{SUMMARY AND DISCUSSION}

We present a theoretical framework for spatial resolution in edge-illumination x-ray tomography, a metric that has specific features as it is decoupled from the source and pixel dimensions due to the structuring of the primary beam into an array of beamlets. Moreover, spatial resolution is also affected by the type of dithering (step-and-shoot or continuous). The option to choose between these different modes of sampling provides flexibility to the user in terms of adjusting the spatial resolution to the specific imaging needs of the scanned sample.

The presented theoretical framework is based on the concept of the bowtie-shaped essential support of the Radon transform in frequency space. The demonstration of the existence of such a support for edge-illumination $\mathrm{x}$-ray tomography and its agreement with the theory are the main results of this article. It is in fact remarkable that a simple model like Eq. 1, based only on two parameters ( $t$ and $w)$ can precisely predict the location of frequency content in 2D Fourier space, despite the fact that the functionality of edge-illumination $\mathrm{x}$-ray tomography generally depends on a variety of additional parameters. In particular, any type of diffusion of the information carried by the individual beamlets, caused by, e.g., cross talk among the detector pixels, excessive source blur, or partial transmittance of the masks' gold layers, can worsen its performance [27]. However, the theory presented in this paper proves to be relatively robust against these imperfections, as shown by the good agreement with the experimental results. It should be noted though that some effort is taken to minimize signal diffusion by employing a photon-counting detector, and that further work would be required to evaluate the model's performance in a scenario where significantly higher levels of cross talk are present.

As a second aspect, we exploit the existence of the essential support in frequency space to derive optimal sampling conditions for edge-illumination x-ray tomography, providing insight on how to acquire data to maximize spatial resolution (at minimal dose). Validating these conditions reveals a limitation of the theory, as in the simulated, noise-free case sampling above the optimal rates provides access to higher spatial frequencies than predicted. This is due to the fact that, while present and significant in the absence of noise, higher-order contributions to spatial resolution are neglected in the theory, which is reasonable when dealing with real (noisy) data. The optimality conditions derived in this article should therefore be considered a practical guide for choosing lateral and angular sampling steps for experimental edge-illumination x-ray tomography scans, rather than a rigorous recipe for predicting the outcome of noiseless simulations.

Higher-order contributions to spatial resolution are most commonly due to oscillations in the frequency response function of a square aperture (that are typically neglected).
It should be noted, however, that in the special case of edge-illumination $\mathrm{x}$-ray tomography higher-order effects can also occur due to other reasons. In fact, contrary to an assumption commonly made as part of the derivation of phase-retrieval algorithms, the image-formation process in this method is not strictly uniform across the beamlet width. As was shown previously [10], the attenuation contrast in a projection originates to a large extent from those parts of the beamlets that are not stopped by the second mask in front of the detector, while the refraction contrast can originate from the entire beamlet cross section. In addition to that, small-angle scattering might occur on a scale below the beamlet width, which can be accessed when the experimental setup is operated in dark-field mode [28]. Both of these features imply that edge-illumination x-ray tomography could ultimately be used for super-resolution imaging on the subbeamlet scale. Generally, when increasing the spatial resolution care has to be taken to minimize errors in the sampling positions due to potential positioning uncertainties of the involved translation and rotation stages.

While the sampling conditions here are derived for slitshaped apertures, extensions to other aperture shapes are possible and will be investigated as part of future work. One focus might be on using round apertures generating pencil beams, which would lead to a decoupling of the spatial resolution from the source and detector blur along other directions than the in-slice plane. We believe that, by designing dedicated sampling schemes, this could be exploited to the full, leading to a highly versatile imaging system with regards to spatial resolution.

Generally, we expect the results presented in this article to inform the design of new experimental setups and/or data acquisition schemes. We also anticipate that it will help to understand how spatial resolution (and hence image quality) is affected when the optimal sampling conditions cannot be met, e.g., due to constraints on scan time or dose.

\section{ACKNOWLEDGMENTS}

C.K.H. and M.E. are supported by the Royal Academy of Engineering under the RAEng Research Fellowship scheme, F.A.V. is supported by the Royal Academy of Engineering and the Office of the Chief Science Adviser for National Security, and A.O. wishes to acknowledge support from the UK Engineering and Physical Sciences Research Council (Grant No. EP/I021884/1).

[1] A. Olivo and R. Speller, A coded-aperture technique allowing $\mathrm{X}$-ray phase contrast imaging with conventional sources, Appl. Phys. Lett. 91, 074106 (2007). 
[2] C. Hagen, P. Munro, M. Endrizzi, P. Diemoz, and A. Olivo, Low-dose phase contrast tomography with conventional $\mathrm{x}$ ray sources, Med. Phys. 41, 070701 (2014).

[3] S. Wilkins, T. Gureyev, D. Gao, A. Pogany, and A. Stevenson, Phase-contrast imaging using polychromatic hard $\mathrm{x}-$ rays, Nature 384, 335 (1996).

[4] D. Chapman, W. Thomlinson, R. Johnston, D. Washburn, E. Pisano, N. Gmur, Z. Zhong, R. Menk, F. Arfelli, and D. Sayers, Diffraction enhanced imaging, Phys. Med. Biol. 42, 2015 (1997).

[5] F. Pfeiffer, T. Weitkamp, O. Bunk, and C. David, Phase retrieval and differential phase-contrast imaging with lowbrilliance X-ray sources, Nat. Phys. 2, 258 (2006).

[6] I. Zanette, T. Zhou, A. Burvall, U. Lundstrm, D. H. Larsson, M. Zdora, P. Thibault, F. Pfeiffer, and H. M. Hertz, SpeckleBased X-Ray Phase-Contrast and Dark-Field Imaging with a Laboratory Source, Phys. Rev. Lett. 112, 253903 (2014).

[7] F. A. Vittoria, M. Endrizzi, P. C. Diemoz, U. H. Wagner, C. Rau, I. K. Robinson, and A. Olivo, X-ray absorption, phase and dark-field tomography through a beam tracking approach, Sci. Rep. 5, 16318 (2015).

[8] F. A. Vittoria, M. Endrizzi, and A. Olivo, Multi-Modal Phase-based Micro-Tomography with Non-Micro-Focal Laboratory Sources, Phys. Rev. Appl. 8, 064009 (2017).

[9] A. Bravin, P. Coan, and P. Suortti, X-ray phase contrast imaging: From pre-clinical applications towards clinics, Phys. Med. Biol. 58, R1 (2013).

[10] P. Diemoz, F. Vittoria, and A. Olivo, Spatial resolution of edge illumination x-ray phase contrast imaging, Opt. Express 22, 15514 (2014).

[11] M. Endrizzi, F. Vittoria, P. Diemoz, R. Lorenzo, R. Speller, U. Wagner, C. Rau, I. Robinson, and A. Olivo, Phasecontrast microscopy at high x-ray energy with a laboratory setup, Opt. Lett. 39, 3332 (2014).

[12] C. Hagen, P. Coan, A. Bravin, A. Olivo, and P. Diemoz, A continuous sampling scheme for edge illumination $\mathrm{x}-$ ray phase contrast imaging, J. Appl. Phys. 118, 054901 (2015).

[13] P. A. Rattey and A. G. Lindgren, Sampling the 2-D Radon transform, IEEE Trans. Acoust. Speech Signal Process. 29, 994 (1981).

[14] N. T. Vo, M. Drakopoulos, R. C. Atwood, and C. Reinhard, Reliable method for calculating the center of rotation in parallel-beam tomography, Opt. Express 22, 19078 (2014).
[15] J. C. Da Silva, M. Guizar-Sicairos, M. Holler, A. Diaz, J. A. van Bokhoven, O. Bunk, and A. Menzel, Quantitative region-of-interest tomography using variable field of view, Opt. Express 26, 16752 (2018).

[16] H. Nyquist, Certain Topics in Telegraph Transmission Theory, Trans. Am. Inst. Electr. Eng. 47, 617 (1928).

[17] C. E. Shannon, Communication in the presence of noise, Proc. IRE 37, 10 (1949).

[18] F. Natterer, The Mathematics of Computerized Tomography (Vieweg+Teubner, Wiesbaden, 1986).

[19] L. Desbat, Efficient sampling on coarse grids in tomography, Inverse Probl. 9, 251 (1993).

[20] D. Potts and G. Steidl, Fourier Reconstruction of Functions from their Nonstandard Sampled Radon Transform, J. Fourier Anal. Appl. 8, 513 (2002).

[21] I. Zanette, M. Bech, F. Pfeiffer, and T. Weitkamp, Interlaced phase stepping in phase-contrast x-ray tomography, Appl. Phys. Lett. 98, 094101 (2011).

[22] F. Vittoria, P. Diemoz, M. Endrizzi, L. Rigon, F. Lopez, D. Dreossi, P. Munro, and A. Olivo, Strategies for fast and efficient wave optics simulation of coded-aperture and other X-ray phase contrast imaging methods, Appl. Opt. 52, 6940 (2013).

[23] M. Endrizzi, F. A. Vittoria, G. Kallon, D. Basta, P. C. Diemoz, A. Vincenzi, P. Delogu, R. Bellazzini, and Alessandro Olivo, Achromatic approach to phase-based multi-modal imaging with conventional x-ray sources, Opt. Express 23, 16473 (2015).

[24] P. Munro, C. Hagen, M. Szafraniec, and A. Olivo, A simplified approach to quantitative $\mathrm{x}$-ray phase imaging, Opt. Express 21, 11187 (2013).

[25] P. C. Diemoz, C. K. Hagen, M. Endrizzi, M. Minuti, R. Bellazzini, L. Urbani, P. De Coppi, and A. Olivo, Single-Shot X-ray Phase-Contrast Computed Tomography with NonMicrofocal Laboratory Sources, Phys. Rev. Appl. 7, 044029 (2017).

[26] D. Paganin, S. C. Mayo, T. E. Gureyev, P. R. Miller, and S. W. Wilkins, Simultaneous phase and amplitude extraction from a single defocused image of a homogeneous sample, J. Microsc. 206, 33 (2002).

[27] K. Ignatyev, P. R. T. Munro, R. D. Speller, and A. Olivo, Effects of signal diffusion on $\mathrm{X}$-ray phase contrast images, Rev. Sci. Instrum. 82, 073702 (2011).

[28] M. Endrizzi and A. Olivo, Absorption, refraction and scattering retrieval with an edge-illumination-based imaging setup, J. Phys. D: Appl. Phys. 47, 505102 (2014). 